\title{
Genomic and transcriptomic analyses reveal a tandem amplification unit of 11 genes and mutations in mismatch repair genes in methotrexate-resistant HT-29 cells
}

\author{
Ahreum Kim ${ }^{1,2,3,5}$, Jong-Yeon Shin ${ }^{1,4,5}$ and Jeong-Sun Seo $1,2,4 凶$ \\ (c) The Author(s) 2021
}

\begin{abstract}
DHFR gene amplification is commonly present in methotrexate (MTX)-resistant colon cancer cells and acute lymphoblastic leukemia. In this study, we proposed an integrative framework to characterize the amplified region by using a combination of single-molecule real-time sequencing, next-generation optical mapping, and chromosome conformation capture (Hi-C). We identified an amplification unit spanning 11 genes, from the DHFR gene to the ATP6AP1L gene position, with high adjusted interaction frequencies on chromosome $5(\sim 2.2 \mathrm{Mbp})$ and a twenty-fold tandemly amplified region, and novel inversions at the start and end positions of the amplified region as well as frameshift insertions in most of the $M S H$ and $M L H$ genes were detected. These mutations might stimulate chromosomal breakage and cause the dysregulation of mismatch repair. Characterizing the tandem gene-amplified unit may be critical for identifying the mechanisms that trigger genomic rearrangements. These findings may provide new insight into the mechanisms underlying the amplification process and the evolution of drug resistance.
\end{abstract}

Experimental \& Molecular Medicine (2021) 53:1344-1355; https://doi.org/10.1038/s12276-021-00668-x

\section{INTRODUCTION}

Gene amplification, the triggering of an abnormal copy number increase in a specific region of the genome of cells growing under a selective condition, is associated with the overexpression of oncogenes such as MYC, MYCN, and ERBB, which engender abnormal cell proliferation and replication ${ }^{1-3}$. These genes undergo amplification much more frequently than simple mutation, since the rate of gene amplification is greater than the mutation rate in mammalian cells ${ }^{4}$; thus, such amplification has great tumorigenic potential $^{5}$.

However, no molecular targeted agents have been specifically developed to prevent gene amplification because of the associated chromosomal complexity and technical limitations. It is therefore critical to find predictive and prognostic biomarkers for gene amplification to develop specific treatments that will improve patient outcomes and optimize therapeutic decisions $s^{6,7}$.

In addition, gene amplification is an indicator of a drug-resistant sample in cancer and healthy cells ${ }^{8}$, so it will be important to identify the genetic features or pathways that promote amplification in tumors. These mechanisms might serve as therapeutic targets that can prevent drug resistance and arrest or eradicate tumor cells ${ }^{9}$. However, the molecular mechanisms that contribute to high gene copy numbers are completely unknown, since sequence alignment and assembly programs that rely on short reads are not equipped to address genomic rearrangements and repetitive sequences ${ }^{10,11}$.
Short reads, which cannot be used to sequence repetitive sites, can only rarely be aligned to the accurate site of reference or the site of an abrupt change in copy number in the gene-amplified region, and previous research findings have proven the poor performance of short-read sequencing for structural variant detection as well as alignment ${ }^{12,13}$.

DHFR gene amplification at chromosome 5 is a hallmark of methotrexate (MTX) resistance in colon cancer cells and acute lymphoblastic leukemia. MTX is an antifolate drug that inhibits dihydrofolate reductase (DHFR) by preventing DNA synthesis and cell division ${ }^{14-16}$.

Amplification of the DHFR gene generates two major DNA segments consisting of extrachromosomal double minutes (DMs) and intrachromosomal homogeneously staining chromosome regions (HSRs); however, the molecular mechanisms underlying the generation of these amplified region products and suitable methods for their detection and characterization are still unknown.

In this study, single-molecule real-time (PacBio SMRT) sequencing, optical genome mapping (BioNano Genomics; read size: $\sim 10 \mathrm{~kb}$ ), and high-throughput chromosome conformation capture $(\mathrm{Hi}-\mathrm{C})$ for inter- and intrachromosomal interactions were used to identify relevant repetitive rearrangements with amplified segments and interpret gene amplification mechanisms in an MTXresistant colon cancer cell line (HT-29), which has a heterogeneously amplified genome.

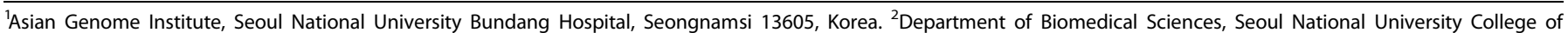

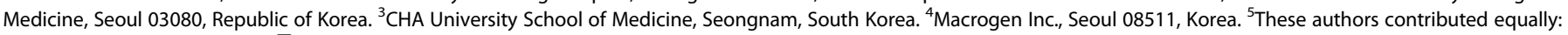
Ahreum Kim, Jong-Yeon Shin. ${ }^{\bigotimes_{e}}$ ail: jeongsun@snu.ac.kr 
These techniques allowed the accurate quantification of amplification size and identification of drastic differences in chromosomal abnormalities and structural variants in MTXresistant compared to MTX-sensitive samples, which are difficult to analyze in detail using short-read sequencing and fluorescence in situ hybridization (FISH). Accordingly, this study contributes to our understanding of basic genomic principles, the impacts of genetic rearrangements on cancer cells, and, by extension, how drug resistance arises from such rearrangements.

\section{MATERIALS AND METHODS Preparation of MTX-resistant cancer cells and sensitization procedure}

After curating a list of cancer cell lines published in previous studies, we targeted human colon adenocarcinoma HT-29 cells. The HT-29 line was obtained from the Korean Cell Line Bank $(\mathrm{KLCL})$ and cultured to develop MTX-resistant cancer cells, as described previously ${ }^{17}$. HT-29 was chosen because it can be engineered to grow in high concentrations of MTX via the amplification of DHFR.

Experimental and computational analyses were performed on a methotrexate-resistant colon cancer cell line (HT-29) to characterize the amplified gene region and understand the underlying mechanism (Supplementary Fig. 1).

To generate MTX-resistant HT-29 cells, we optimized the MTX concentration and cultured the cells in RPMI media supplemented with $10 \%$ fetal bovine serum and graded concentrations (from $10^{-8}$ to $10^{-6} \mathrm{M}$ ) of MTX in five T25 flasks per cell line. The MTX solution was prepared by mixing $100 \mathrm{mg}$ MTX powder with $1.967 \mathrm{ml}$ DMSO. This mixture was then aliquoted at $100 \mu \mathrm{l} / \mathrm{ml}$ and kept at $-20^{\circ} \mathrm{C}$. A limited number of resistant cells $\left(\sim 3 \times 10^{5}\right)$ from each T25 flask were plated in Petri dishes in the same culture medium and MTX concentration.

We then used $3.2 \mathrm{~mm}$-diameter cloning discs (Sigma) to transfer 3 isolated clones (C1-2, C4-3, C8-22) from each Petri dish to another T25 flask and then cultured these cells under the same conditions. Subsequently, the MTX-resistant clones were passaged 40 times without MTX. For the second and third treatment cycles, $3 \times 10^{5}$ clones and parental HT-29 cells were cultured in $25 \mathrm{~cm}^{2}$ flasks. A stepwise increase in MTX concentration from $10^{-8} \mathrm{~mol} / \mathrm{L}$ to $10^{-6} \mathrm{~mol} / \mathrm{L}$ was applied; MTXresistant cells were grown in $10^{-6} \mathrm{~mol} / \mathrm{L}$ MTX.

\section{Detection of gene amplification}

The copy number of MTX-resistant clones was measured via TaqMan Copy Number Reference Assays and Viia7 technology to select clones with high $D H F R$ gene amplification. The DHFR gene copy number was estimated via relative quantitation ( $\mathrm{RQ})$ using the comparative $\mathrm{Ct}$ method and computed using the $\mathrm{Ct}$ difference (delta $\mathrm{Ct}$ ) between MTX-resistant samples and reference samples (HapMap NA19982). Then, the $\Delta C t$ values of MTXresistant samples were compared to those of a HapMap reference sample known to have two copies of DHFR, such that they were two times the relative quantity of the reference. The following three equations were used to compute the copy number from the $\mathrm{Ct}$ value. The statistical significance was computed by the R package ' $p c r$ ') as described in the package instructions for MTX-resistant HT-29 samples.

Expression $=2^{-}(($Sample $\mathrm{Ct}-\Re$ ference $\mathrm{Ct})-($ Control $\mathrm{Ct}-\Re$ ference $\mathrm{Ct}))$

Rate between reference and sample $=\frac{\text { Expression for Sample }}{\text { Expression for Reference }}$

Copy number $=2$ copies (reference) $\times$ Rate

Samples with a high DHFR copy number were selected for FISH and karyotyping to visualize and map $D H F R$ and detect chromosomal abnormalities. The DHFR red signal and $5 \mathrm{p} 12$-green signal were counted at anaphase and metaphase, with $\times 1000$ magnification and a three-color (RGB) filter.

\section{RNA-seq analysis and transcriptome profiling}

RNA-seq of MTX-resistant HT-29 cells and control samples was performed to investigate expression profiling. Total RNA, with ribosomal RNA removed, was prepared and sequenced using the Illumina HiSeq 2000 system (depth 100X), as previously described ${ }^{18}$. The obtained reads were mapped to the human reference genome (GRCh38) using the Spliced Transcripts Alignment to a Reference (STAR) tool to produce analysis-ready BAM files (Supplementary Table 1). We followed the key principles of processing and analysis steps from the GATK website. The mapped reads (BAM file) were visualized via SeqMonk.

To estimate the expression of each gene, raw reads were counted using the HTSeq-count tool and normalized to variance stabilizing data (VSD) expression via the $\mathrm{R}$ package DEseq2. Fragments per kilobase million (FPKM) values were calculated using the $R$ package edge $R$ and converted to $\log _{2}$ values. The median-centered gene expression was computed from FPKM expression using Cluster 3.0 software, which subtracts the row-wise median from the expression values in each row. The median-centered VSD and FPKM expressions were visualized in a heatmap.

\section{Variant discovery analysis}

Variant calling was performed on transcriptome datasets. The duplicated sites from analysis-ready BAM files were filtered with Picard, and variants were called and filtered by removing spurious and known RNA-editing sites in VCF format. The genomic variants in the control and MTX-resistant HT-29 samples were compared. We performed variant discovery analyses according to the step-by-step recommendations provided by the Genome Analysis Toolkit (GATK) to obtain high-quality variants ${ }^{19}$.

To determine the exact SNPs from the call set, the variants were filtered out according to several criteria. First, the cutoff for quality-by-depth (QD) was 3.0, which is the variant confidence score divided by the unfiltered depth of coverage. Variants were filtered out if they had a score $<3.0$. Second, the variants were filtered out when the Fisher strand (FS) score was $>30.0$, which indicated the Phred-scaled $p$-value using Fisher's exact test for detecting strand bias $^{20}$. The identified and filtered variants were annotated using RefSeq genes and the ANNOVAR tool.

\section{Differentially expressed gene analysis}

Differentially expressed genes (DEGs) between MTX-resistant HT-29 cells and control samples were analyzed using the R packages DESeq 2 and edgeR ( $P$-value $<0.05$, Log2 (fold change) $\mid \geq 1$, and baseMean $\geq 100$ ). The DEGs were then subjected to enrichment analysis with KEGG gene sets via Gene Set Enrichment Analysis (GSEA).

\section{Alternative splicing event analysis}

The exon inclusion levels, defined with junction reads from RNA sequencing results, were subsequently processed via rMATS.3.2.5.21. Five different types of alternative splicing events (SE: skipped exon, MXE: mutually exclusive exon, A5SS: alternative $5^{\prime}$ splice site, A3SS: alternative $3^{\prime}$ splice site, RI: retained intron) were identified in both MTX-resistant HT-29 cells and control samples. The number of significant events was detected using both junction counts and on-target reads.

\section{PacBio long-read sequencing analysis}

Genomic DNA was extracted from MTX-resistant HT-29 cells and control samples using the Gentra Puregene Cell kit (Qiagen), and libraries were constructed for PacBio sequencing. The PacBio long reads were aligned to the human genome (version GRCh38) with BWA-mem aligner, and the preprocessing pipeline on the BWA-mem website was followed to ensure the technical and biological quality of the results (Supplementary Table 2). The read depth was estimated via the depth-of-coverage option in GenomeAnalysisTK. Regions with coverage differences of $>10 X$ between MTX-resistant HT-29 cells and controls were selected as amplified regions.

\section{Detection of genomic variants and amplification units}

The structural variants (deletion, duplication, inverted duplication, translocation, and inversion) in MTX-resistant HT-29 cells and control samples were analyzed from sorted PacBio output derived from BWA-MEM using Sniffles ${ }^{22}$. The BAM files were converted to binned copy numbers across a genome using Copycat. The genomic rearrangements were visualized from VCF files (Sniffles) and read coverage files (Copycat) via SplitThreader (http://splitthreader.com/12. BWA-MEM and Sniffles were used in combination to successively scan the alignments to identify all types of SVs in tandem gene-amplified regions, including repeat-rich regions and complex nested events ${ }^{23}$. 


\section{Long-read sequencing and optical genome mapping}

The long-read sequencing coverage was designed to be $\sim 10 \mathrm{X}$, and the coverage was not sufficient to perform de novo assembly except in the tandemly amplified region ( 197X). Thus, only the 54,804 PacBio reads from our tandemly amplified region were de novo assembled using the $\mathrm{PBCR}$ assembler, and 10 contigs were generated by using the previously reported method ${ }^{24}$. Of these, 6 contigs were used for reference-assisted genome ordering utility (Ragout) with hg 38, and we had one scaffold from 6 contigs. The other contigs were alternatively assembled and finally matched with other contigs. In addition, PacBio PBCR contigs were compared with the Bionano contigs to identify the accuracy of assembly (Supplementary Tables 3 and 4).

Optical mapping of the PacBio assembly data was performed using BioNano Assembler software (Irys System, BioNano Genomics) to obtain accurate sequences. High molecular weight DNA was isolated using the IrysPrep Plug Lysis Long DNA Isolation Protocol (Bionano Genomics). In brief, cells were trypsinized, washed in FBS/PBS, counted, rewashed in PBS, and embedded in agarose plugs using components from the Bio-Rad Plug Lysis Kit. The plugs were subjected to proteinase $\mathrm{K}$ digestion $(2 \times 2 \mathrm{~h}$ at RT). After a series of washes in the buffer from the Bio-Rad kit, followed by washes in TE (Tris-EDTA), the plugs were melted and treated with GELase enzyme (Epicenter).

High molecular weight DNA was released and subjected to drop dialysis. The DNA was left to equilibrate for 4 days and then quantified using the Qubit Broad Range dsDNA Assay Kit (Thermo Fisher Scientific). Using the IrysPrep NLRS assay (Bionano Genomics), $200-300 \mathrm{ng} / \mathrm{\mu L}$ of high molecular weight DNA underwent single-strand nicking with 10 units of Nt.BspQI nickase (New England BioLabs). Nicked sites were repaired with fluorophore-labeled nucleotides to restore strand integrity.

The backbone of the fluorescently labeled double-stranded DNA was stained with the intercalation dye YOYO-1. Labeled molecules were loaded directly onto IrysChip without further fragmentation or amplification and imaged using the Irys instrument. Multiple cycles were performed to reach an average raw genome depth-of-coverage of 50X. In addition, the tandem repeats in the amplified region in both the assembly and raw data were identified using IrysView 2.0 software (Supplementary Tables 5 and 6).

\section{Hi-C data analysis}

Approximately 50 million MTX-resistant HT-29 cells and control cells were used to produce high-throughput chromatin conformation capture ( $\mathrm{Hi}-\mathrm{C})$ datasets. We generated $2 \mathrm{Hi}-\mathrm{C}$ libraries using the Hindlll restriction enzyme, following a previously established protocol ${ }^{25}$. In brief, the $\mathrm{Hi}-\mathrm{C}$ protocol involves crosslinking cells with formaldehyde, permeabilizing them while keeping the nuclei intact, digesting the DNA with a suitable restriction enzyme, filling the $5^{\prime}$-overhangs while incorporating a biotinylated nucleotide, ligating the resulting blunt-end fragments, shearing the DNA, capturing the biotinylated ligation junctions with streptavidin beads, and analyzing the resulting fragments with paired-end sequencing via $\mathrm{Hi}-\mathrm{Seq} 2000$.

The $\mathrm{HiC}$ data (fastq files) were processed to normalize the contact matrices using HiC-Pro version 2.10.0 ${ }^{26}$. The pipeline was based on the Bowtie 2 aligner, and the selected restriction enzyme (HindIII) was used to generate normalized contact maps, as described in the HiC-Pro pipeline (https://github.com/nservant/HiC-Pro). Each aligned read was assessed to determine the valid interactions and control quality by excluding invalid ligation products and duplicated valid pairs (Supplementary Table 7). The aligned $\mathrm{Hi}-\mathrm{C}$ sam files were converted into the HiCnv format, which calls CNVs from Hi-C data ${ }^{27}$. In addition, interchromosomal translocations and their boundaries were detected from a $\mathrm{Hi}-\mathrm{C}$ matrix file using $\mathrm{HiCtrans}$. The list of valid interaction output files called by $\mathrm{HiC}$-pro was converted to a Juicebox input file and visualized using Juicebox (https://github.com/ theaidenlab/juicebox/wiki).

The $\mathrm{R}$ package HiCcompare was used to detect differential spatial chromatic interactions on a genome-wide scale between control and MTXresistant HT-29 cells $^{28}$. Using this package, the interaction frequencies after adjustment with joint-normalization, adjusted $p$-values, and filtered lowaverage expression after multiple testing correction were determined.

To remove background interactions from the inter- and intrachromosomal interactions, we compared chromosomal interactions between the control and MTX-resistant samples by considering the combination of eigenvector, balanced value, and coverage, as well as the interaction frequencies after adjustment with joint normalization. $\mathrm{Hi}-\mathrm{C}$ contact maps were visualized in Juicebox with applied balanced normalization and eigenvector and coverage. We calculated the eigenvector (first principal component) of the Pearson correlation of the (binned) HiC contacts and annotated contact domains by using the arrowhead option, and balanced normalization (Knight-Ruiz) was used to remove background.

\section{Statistical tests}

All statistical analyses were performed using R-3.3.0. The gene expression levels in MTX-resistant and control HT-29 cells were compared, and the $p$-value was determined using unpaired Student's $t$-test or Mann-Whitney $U$-test, based on the Shapiro-Wilk normality test. $P$-values $<0.05$ were considered to be statistically significant.

\section{Transposable element detection}

Transposable elements (TEs) were identified and compared between control and MTX-resistant HT-29 cells by using Censor, which is a program for the detection of $\mathrm{TEs}^{29}$.

\section{RESULTS}

\section{Analysis of MTX-resistant HT-29 cells}

While generating MTX-resistant HT-29 cells (selected clone: C1-2) from single-cell selection and MTX sensitization as previously described ${ }^{30}$, dramatic morphological changes in the cells themselves were observed; rounded and circular cell shapes were observed during the first cycle of sensitization, and rod and irregular shapes were seen during the second cycle (Supplementary Fig. 2). The original shapes were again observed during the third cycle of sensitization, which might indicate that HT-29 cells became resistant to MTX and grew rapidly under high MTX concentrations.

As expected, DHFR expression, which was normalized to the expression of the $B 2 M$ housekeeping gene, steadily increased from the first to third cycle, as the HT-29 clones became resistant to MTX (Supplementary Fig. 3a). After confirming these morphological changes and increased DHFR expression, the DHFR copy number was measured in several clones.

The cycle quantification values $(\mathrm{Ct})$ of the clones were used to compute the rate of copy number change. These values dropped from 26.04 to 19.99 as the number of cycles increased (estimate: 4.075, $p=0.003758396,[95 \% \mathrm{Cl}]=1.8-6.3$, Supplementary Table 8). Interestingly, there was a dramatic increase in the DHFR copy number between the first ( 0.97 copies) and second (54.83 copies) cycles (Supplementary Fig. 3b).

Based on these results, we ascertained that a specific time period and set of conditions were required for clones to survive in the presence of MTX, and amplification of the DHFR gene was an indicator of MTX resistance, as previously described ${ }^{14}$.

\section{Validation of gene amplification in MTX-resistant HT-29 cells} After quantifying the DHFR gene copy number, the amplification of the DHFR gene at the $5 \mathrm{q}$ arm was visualized via fluorescent in situ hybridization (FISH) of an MTX-resistant clone (C1-2) and control sample. The DHFR gene regions on the $5 q$ arm of cells in the second and third cycles were abnormally long compared to that of the control, as expected (Supplementary Fig. 4).

The amplified DHFR gene patterns of C1-2 cells in metaphase revealed that the amplified region had a chromosome painting signal, and the FISH signal patterns were highly heterogeneous. In total, 9 minor signal-amplified patterns and two major patterns accounted for 44 and $28 \%$ of abnormal DHFR gene amplification patterns, respectively (Supplementary Fig. 5).

The optimized C1-2 clone (C1-2-4), which was robust in the presence of MTX, was visualized using FISH. Four different types of gene amplification patterns were detected: two patterns had amplified DHFR genes at two q arms (75 and 12.5\%), one pattern had amplified DHFR genes at three q arms (i.e., an isochromosome pattern) $(8.3 \%)$, and one completely lacked amplified DHFR genes at q arms (4.2\%) (Fig. 1). Overall, $\sim 96 \%$ of the cells had at least one DHFR-amplified region. 
Control (HT-29)

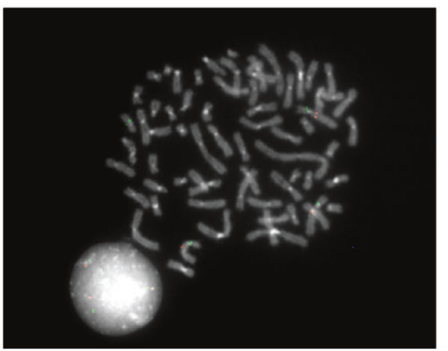

FISH

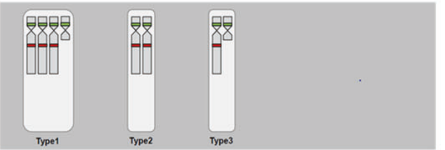

$33.3 \% 33.3 \% 33.3 \%$

FISH signal type

Metaphase

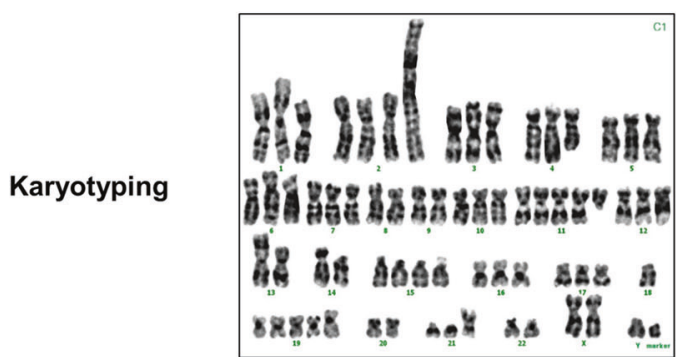

$3^{\text {rd }}$ cycle

MTX resistant HT-29
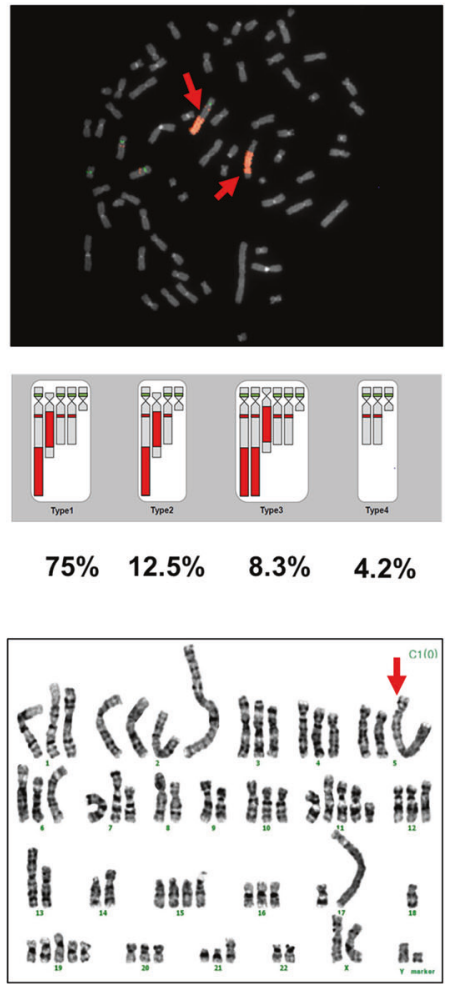

Fig. 1 Visualization of DHFR amplification patterns using FISH and karyotyping of MTX-resistant clones (C1-2-4) and controls. A subculture (C1-2-4) of the C1-2 clone was investigated via fluorescent in situ hybridization (FISH) at $\times 1000$ magnification, and the FISH signal type and percentage at metaphase were compared between controls and MTX-resistant clones (C-1-2-4). All chromosomes were karyotyped, and abnormal chromosomal shapes were detected for the $5 q$ and $17 q$ arms of MTX-resistant cells compared to controls, as indicated by the red arrow.

A majority of these strains were resistant to MTX and exhibited a high level of DHFR gene amplification at the $5 q$ arm. However, the MTX-resistant C1-2-4 cells had a variety of DHFR gene amplification patterns and copy numbers, as well as heterogeneous genetic status in the presence of MTX, even though the strain was generated from a single MTX-resistant cell ${ }^{31}$.

In addition, MTX-resistant HT-29 and control samples were karyotyped to accurately detect the amplified region. The C1-24 clone had a homogeneously stained region (HSR) at the chromosome $5 \mathrm{q}$ arm, as previously described ${ }^{32}$, in addition to an abnormally long $17 q$ arm (Fig. 1). It was previously found that chromosome $17 q$ arm amplification could occur because of the overall genetic instability and karyotype diversity in colorectal cancer $^{33}$.

\section{Analysis of the structural variants and amplification units}

After confirming the HSR on the amplified DHFR region, the five genomic structural variants (deletion, duplication, inverted duplication, translocation, and inversion) and amplified units of MTX-resistant cells were analyzed to identify which genes and structural variants were involved in gene amplification. The $\log _{2}$ ratio of segment coverage over whole chromosomes between the control and MTX-resistant samples was compared. High segment coverage in the MTX-resistant sample, compared to that of the control sample, was observed on chromosome 5 (Fig. 2a). The genes for which the segment coverage was larger than 20 were identified and annotated by position to identify the exact amplified region that included the DHFR gene.

The total number of genomic variants in the MTX-resistant sample was larger than that in the control, and the size of variants such as duplicates and inversions was larger in the MTXresistant sample, which had a large number of split reads (Fig. 2b, c). In addition, more structural variants were detected in MTX-resistant HT-29 cells than in control cells (Supplementary Data 1 and 2).

Selected novel structural variants on chromosome 5 were compared between control and MTX-resistant HT-29 cells. One duplication (median size of split reads: 371,675$)$, three inversions (median size of split reads: 328,697), and three inverted duplications (median size of split reads: 1529) were detected in the MTX-resistant HT-29 cells, while no such variants were detected in the control sample (Table 1). Moreover, the number of split reads for the MTX-resistant HT-29 cells was larger than the average coverage (10X), and most copy number variation (CNV) categories matched, which indicated that the detected structural variants had been accurately detected (Supplementary Table 9 and Supplementary Data 3). Finally, although the number of translocations on chromosome 5 was decreased in the MTXresistant sample, there were more detected interchromosomal genomic rearrangements in the MTX-resistant HT-29 sample (Supplementary Fig. 6).

The position of the amplified region on the chromosome $5 q$ arm was between $80 \mathrm{Mb}$ and $83 \mathrm{Mb}$, which involved the DHFR gene as the start point and included the ATP6AP1L gene. The segment coverage was $\sim 197 \mathrm{X}$, between $80.6 \mathrm{Mb}$ and $82.8 \mathrm{Mb}$ $(\sim 2.2 \mathrm{Mb})$ (Fig. 3a and Supplementary Fig. 7). The amplified region was 20 -fold longer in the MTX-resistant sample than in the control. This size was inferred from the long-read sequencing coverage, which was $10 \mathrm{X}$ in the control sample but abnormally high $(\sim 197 X)$ in only this region in the MTX-resistant sample. 


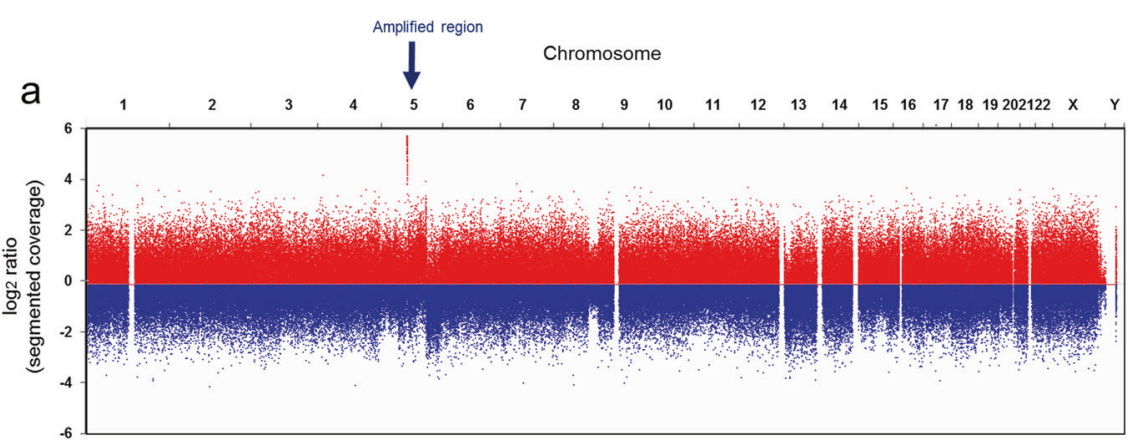

b

C
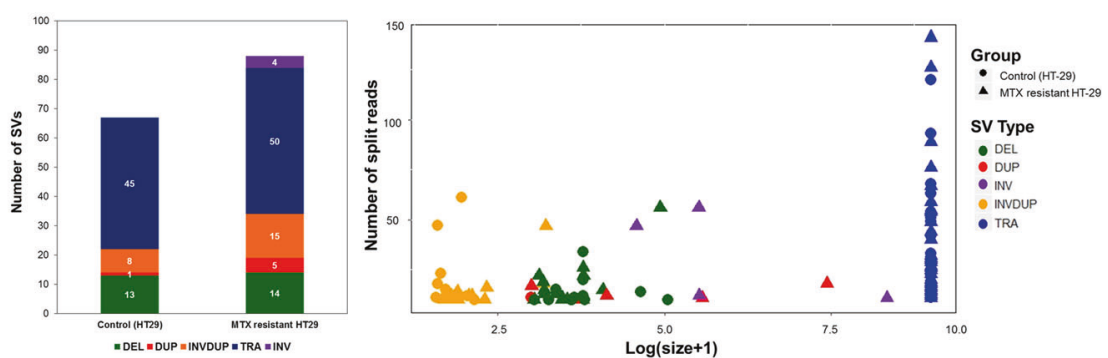

Fig. 2 Detection and characterization of SVs in MTX-resistant HT-29 cells. a The log2 ratio of segment coverage across all chromosomes was compared between control and MTX-resistant HT-29 cells. The amplified region on $5 q$ in MTX-resistant HT-29 cells is indicated by the blue arrow. b Five genomic variants (deletion, duplication, inverted duplication, translocation, and inversion) in the MTX-resistant sample and control were analyzed and visualized in bar graphs, which show the number of variants counted for each sample. $\mathbf{c}$ In the scatter plot, the size of variants $(\log (\operatorname{size}+1))$ and depth of split reads were plotted for control and MTX-resistant HT 29 cells. The group and variant type are indicated by a different shapes and colors.

Table 1. Comparison of detected structural variants between control and MTX-resistant HT-29.

\section{No. of events (median size)}

Deletion

Duplication

Inversion

Inverted duplication

Translocation
All chromosomes

Control (HT-29)

13 (4472)

1 (1004)

0

8 (47)

45
Chromosome 5

MTX-resistant HT-29

14 (2917)

5 (13534)

4 (331155.5)

15 (77)

50
Control (HT-29)

2 (4425.5)

0

0

0

4 (chr3 to chr5) and (chr5 to chr12)
MTX-resistant HT-29

3 (1514)

1 (371675)

3 (328697)

3 (1529)

2 (chr5 to chr12)

The structural variants (deletions, duplications, inversions, inverted duplications, and translocations) were categorized by using Sniffles from sorted PacBio output derived from BWA-MEM over all chromosomes and chromosome 5 in control (HT-29) and MTX-resistant HT-29 cells. The median size of split reads is shown in parentheses.

Overall, the amplification regions included tandem gene amplifications of 11 genes in this region, from DHFR to ATP6AP1L, and involved inversions or inverted duplications at the end of the amplification unit. The tandem duplications of several genes on chr5 (2.2 Mbp) were initiated and terminated by inversion on the specific sequence. The inversion identified at the start point (chr5: $80,618,750-80,631,490$ ) of the amplification unit was evidenced by the three-dot plots compared to hg38. The inverted region included four genes, LINC01337, DHFR, CTC-325J23.2, and MTRNR2L2, and the identified inversion was confirmed by PacBio alignment; however, but other structural variants were not detected because of the difficulty and poor performance of assembly (Supplementary Figs. 8 and 9).

\section{Optical genome mapping over the amplified region}

The region of tandem amplification (80.6-82. Mbp) on chromosome 5 was further analyzed using BioNano genome optical mapping, as good coverage and mapping over the whole range of the amplified region could not be achieved using other methods.
The genome mapping contigs mapped in complex ways to whole chromosomes, except for the amplified region, for which there was high coverage of $\sim 200 X$ (Supplementary Fig. 10). In addition, the gene-amplified unit had inversions at both the start and endpoints of the amplified region, as expected, and there was a newly identified insertion at the endpoint of the amplified region (Fig. 3b).

These inversions were certainly associated with the amplification mechanism and seemed to assist with and even initiate tandem repeat amplification, as previously reported ${ }^{34}$. The identified inverted repeat could stimulate the formation of a large DNA palindrome after the breakage of an adjacent DNA double-strand ${ }^{35}$.

\section{Gene expression levels in the MTX-resistant sample}

There was extremely high gene expression within the identified amplification region, consistent with the high coverage of the amplified unit in our long-read sequencing data (Supplementary Fig. 11 and Supplementary Table 10). The read coverage from 
a
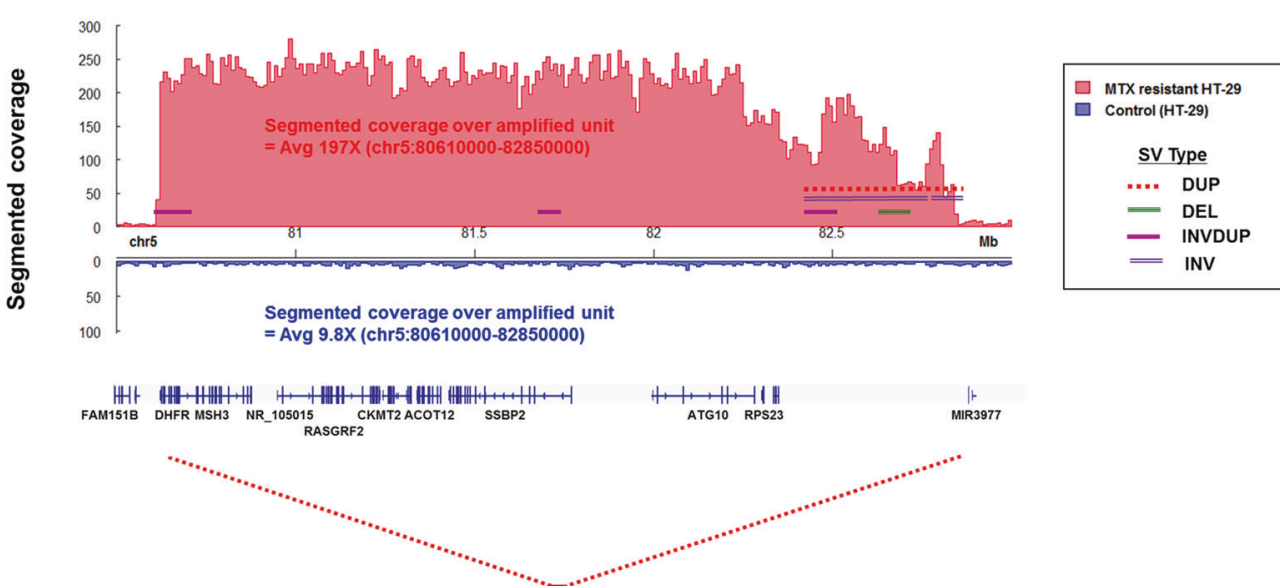

Control (HT-29) TIIDOCIIIIIIII IIID

Twentyfold increase in the amplified unit

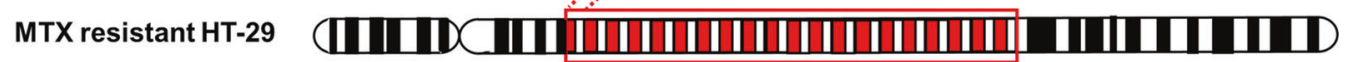

Chromosome 5
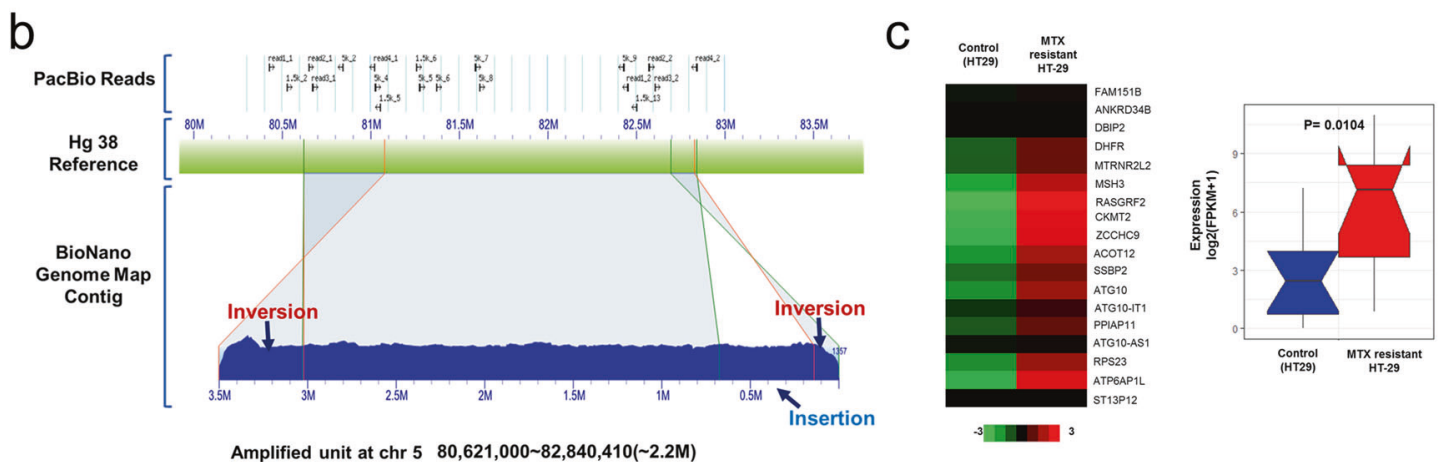

Fig. 3 Detection of amplified units and structural variants over the amplified region in MTX-resistant HT-29 cells. a The segment coverage and genomic variants across the amplified region on chromosome $5(80,610,000-82,850,000)$ were compared between control and MTXresistant HT-29 cells. b The BioNano contig and PacBio long reads matched up with the reference (hg38); several genomic rearrangements are indicated by blue arrows. c The heatmap depicts the gene expression (FPKM) from the amplified units for MTX-resistant HT-29 cells and controls. High expression in a specific region is indicated by a red arrow. The statistical significance ( $p$-value) was computed using the Mann-Whitney test.

long-read sequencing of the amplified region in the MTX-resistant sample was $\sim 10$ times higher than that of the control. Similarly, the $\log _{2}(\mathrm{FPKM}+1)$ expression level from the DHFR to ATP6AP1L gene in MTX-resistant HT-29 cells was $5 X$ that of the control for $D H F R$ and $122 X$ that of the control for RASGRF2 $(P=0.0104$ via the Mann-Whitney test; Fig. 3c).

\section{Identifying novel mutations and their impacts on gene amplification}

To identify relevant mutations in the amplification mechanism, single-nucleotide variants (SNVs) in both samples were identified using transcriptome sequencing data. There were more total exonic mutations across all chromosomes in MTX-resistant HT-29 cells $(13,982)$ than in the control sample $(13,310)$, and on chromosome 5 specifically, 18 more exonic mutations were detected in MTX-resistant HT-29 cells than in controls (Supplementary Tables 11 and 12 and Supplementary Data 4 and 5).

After filtering out synonymous SNVs, we observed that there were several more nonsynonymous mutations (nonsynonymous SNVs, frameshift deletions, frameshift insertions, stop-gains, and stop-losses) in MTX-resistant HT-29 cells compared with control cells across all chromosomes (Supplementary Fig. 12). Some nonsynonymous mutations came from chromosome 5, in which the number and percentage of frameshift insertions were noticeably higher in the MTX-resistant sample (18.1\%) than in the control (4.3\%) (Supplementary Fig. 4a). The most frequently inserted nucleotides in frameshift insertions were thymine (73\%) and adenine (23\%).

This imbalance might explain why the detected frameshift thymine and adenine insertions in mRNA on chromosome 5 cooccurred with DHFR gene amplification, which conferred an ability to survive MTX exposure. Moreover, novel frameshift insertionseither adenine or thymine-were found within the MSH3 and MSH6 genes as well as the PMS1 and PMS2 genes in the MTXresistant HT-29 sample only (Supplementary Table 13). The expression of these genes, except for MSH3, was decreased in the mutated and MTX-resistant HT-29 cells compared to the control sample (Fig. 4b).

The MSH3 and MSH6 genes belong to the mismatch repair $(M M R)$ gene family and are known to play an important role in 
a

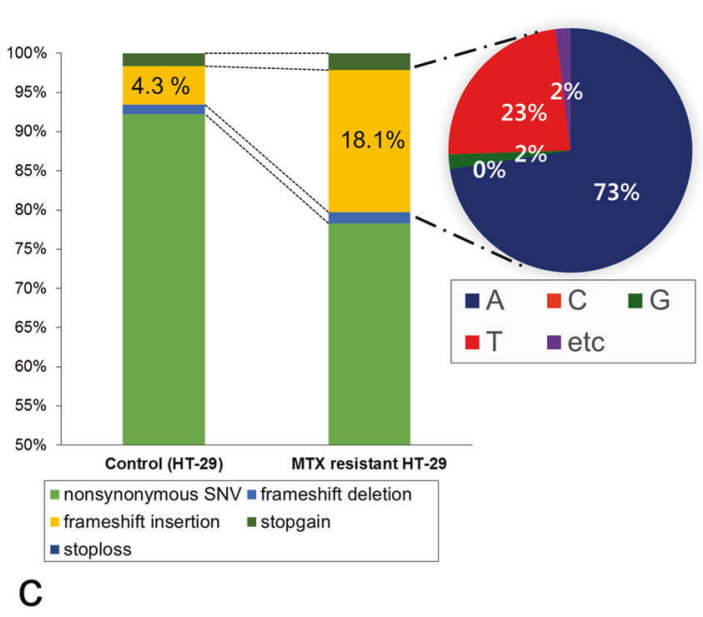

KEGG pathway of down-regulated DEGs $(\mathbf{n}=\mathbf{2 8 7})$ b

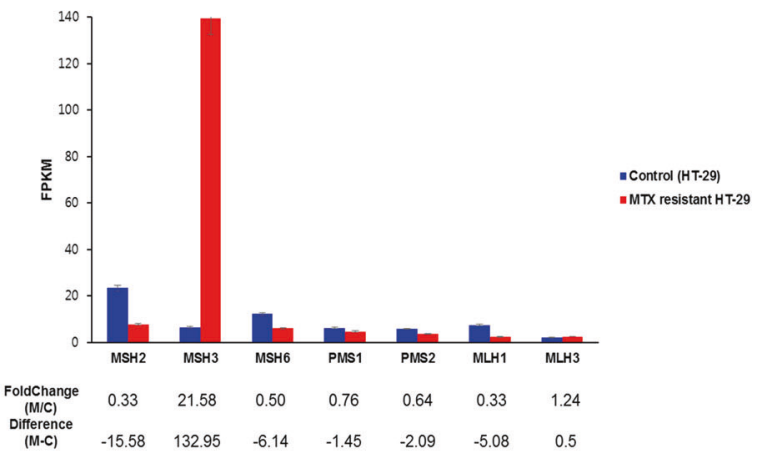

\section{\# of involved genes}

\section{KEGG pathway \\ of up-regulated DEGs $(n=383)$}

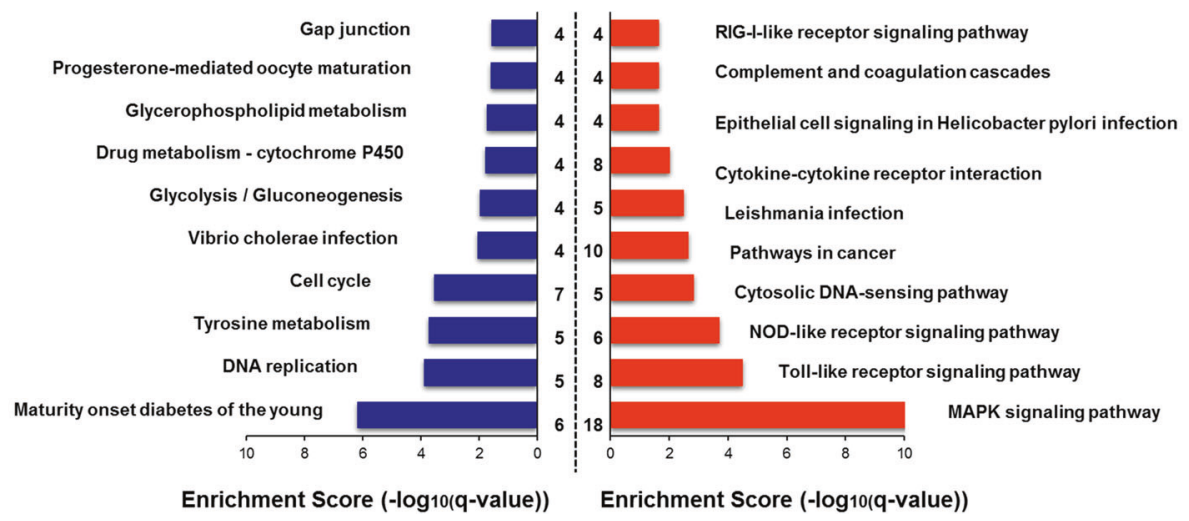

Fig. 4 Comparison of nonsynonymous mutations and $M M R$ gene expression levels. a Nonsynonymous mutations in chromosome 5 (nonsynonymous SNV, frameshift deletion, frameshift insertion, stop-gain, and stop-loss) were compared between MTX-resistant HT-29 cells and control cells. The proportions of the four nucleobases in the frameshift insertions in the MTX-resistant HT-29 cells are indicated. $\mathbf{b}$ Gene expression (FPKM) of mutS homologs and mutL homologs was computed and compared. The changes and differences between MTXresistant HT-29 cells (M) and control cells $(\mathrm{C})$ are indicated below the bar graph. c The top 10 enriched KEGG gene sets among upregulated and downregulated differentially expressed genes are shown according to enrichment score $(-\log (q$ value $)$ ).

DNA repair during cell division as well as in cooperatively suppressing intestinal tumors ${ }^{36}$. MLH (PMS1) and MSH (MSH1 - 6) gene function is significantly correlated with colon cancer; mutations in these genes could cause predisposition and susceptibility to Lynch syndrome, in conjunction with colon cancer $^{37-39}$.

Therefore, novel frameshift insertions in these genes could prevent mismatch repair functionality and tumor suppression in the presence of MTX and stimulate the rapid progression of gene amplification and MTX resistance. The molecular explanation for this tandem gene amplification mechanism could be a malfunction of the MMR pathways ${ }^{40}$. It was previously shown that MSH3 is concurrently amplified with the DHFR gene in MTXresistant cells due to their proximity to each other. An imbalance in the expression of mutS homologs could result in the malfunctioning of base-base mismatch repair and cause genetic instability as well as confer resistance to the cytotoxic effects of MTX ${ }^{41}$.

\section{Identifying DEGs}

To determine which genes and gene sets are involved in MTX resistance, we analyzed DEGs and enriched gene sets (Fig. 4c). A total of 383 upregulated and 287 downregulated DEGs were identified in MTX-resistant HT-29 cells compared to the control sample (Supplementary Table 14).

KEGG enrichment analysis of gene sets was also performed. The DEGs upregulated in MTX-resistant HT-29 cells included IL1B, MAPK11, JUN, MAP3K8, IL8, and CASP1. The affected signaling pathways included MAPK, Toll-like receptor, and NOD-like receptor signaling. Interestingly, the downregulated DEGs, such as MAD2L1, CCNA2, MCM2, MCM4, FEN1, and CDK6, were enriched in DNA replication, tyrosine metabolism, and cell cycle pathways, which are commonly upregulated in colon cancer ${ }^{42}$.

It was previously reported that the DEGs downregulated in MTX-resistant osteosarcoma cell lines were enriched in the mitotic cell cycle, cell cycle, and DNA replication pathways, and these pathways were also downregulated in our MTX-resistant colon cancer cells. This result might explain the role of MTX in inhibiting DHFR and preventing tumor cells from proliferating in both cases ${ }^{43,44}$. In addition, downregulation of MMR gene expression, which affects G2/M cell cycle arrest and apoptosis, might prevent proper checkpoint and cell death signaling; therefore, this phenomenon could contribute to the malfunctioning of DNA replication ${ }^{45}$. 
a

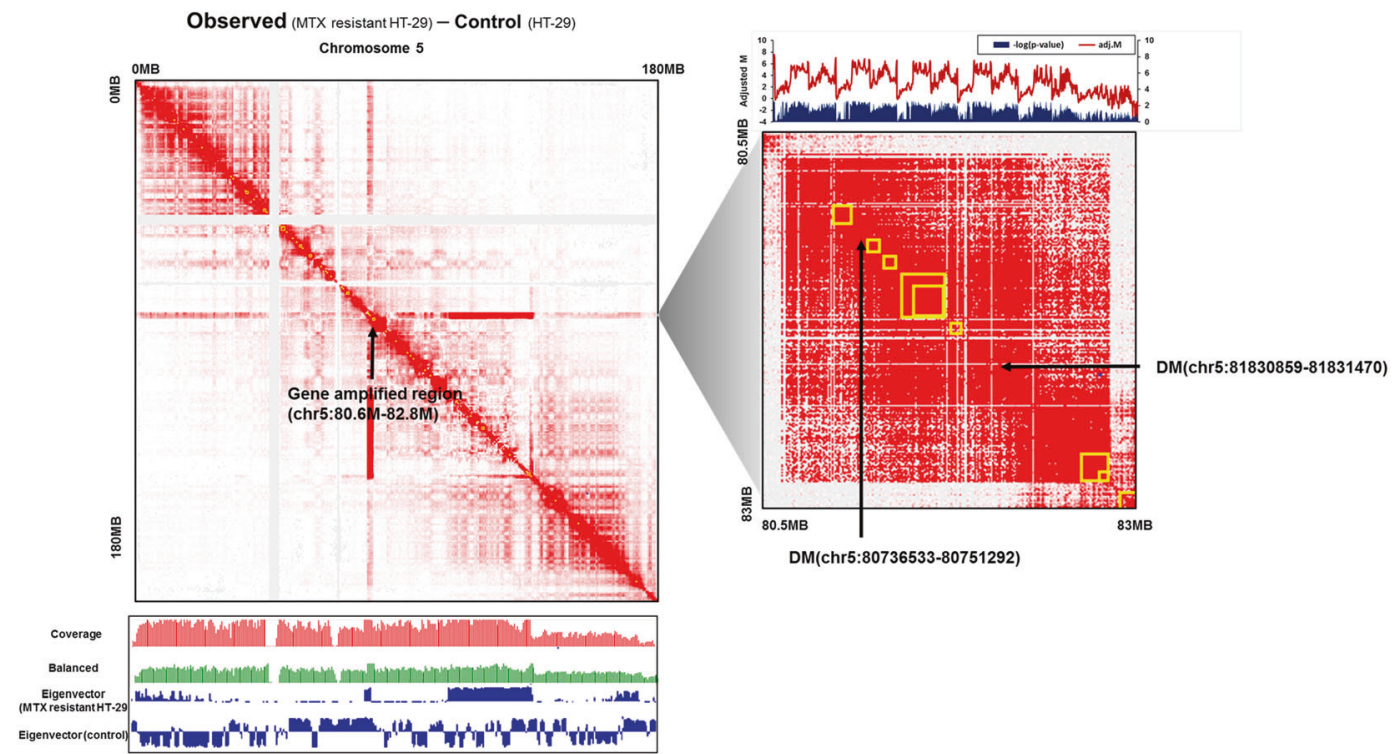

b

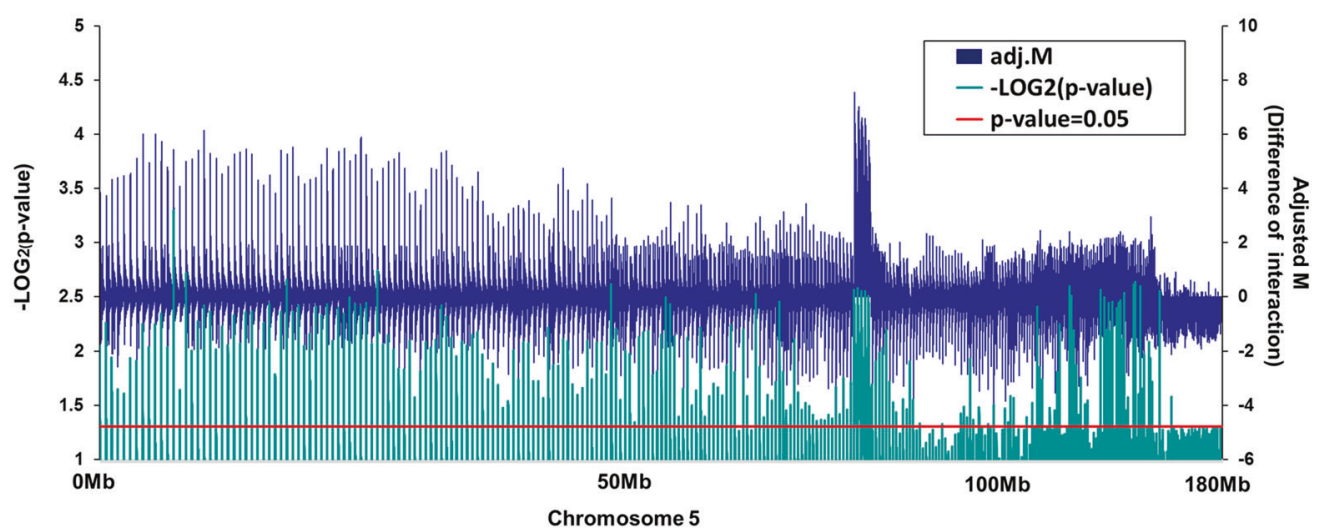

Fig. 5 Topologically associating domains on chromosome $\mathbf{5}$ and adjusted interaction frequencies. a Intrachromosomal interactions for chromosome 5 (MTX-resistant HT-29 - control) are visualized with the coverage and eigenvectors (left), and the newly identified topologically associating domains (TADs) on the duplicated region are indicated by a yellow box at $500 \mathrm{~kb}$ resolution (right). $\mathbf{b}$ The difference in adjusted interaction frequencies (adjusted M) between MTX-resistant and control samples is plotted on a -log2 scale ( $p$-value). Statistical significance $(p$-value $=0.05)$ is indicated by the red line.

\section{Chromosomal interactions and topologically associating domains (TADs)}

Genome-wide intrachromosomal interactions were identified in MTX-resistant HT-29 cells and controls at $5 \mathrm{~kb}$ resolution and compared (Supplementary Fig. 13). A high interaction frequency was apparent, with a clear long red line, on the amplified region only ( 5 q14.1 to $5 q 14.2)$ in the MTX-resistant HT-29 cells. This interaction pattern was similar to those observed for amplification regions in tumor samples in a previous study ${ }^{46}$ (Supplementary Fig. 14).

The TADs and several chromosomal rearrangements at chromosome 5 were identified to visualize the conformations and intra- or interchromosomal interactions within the amplified region and to detect unforeseen chromosomal rearrangements at $500 \mathrm{~kb}$ resolution (Fig. 5a). Frequent intrachromosomal interactions were observed in the amplified region (chr5: 80.6-82.8 Mb), and there were several newly identified TADs in the middle and endpoint of this region compared to those of the control with high adjusted interaction frequencies (adjusted $\mathrm{M}$ ) and an adjusted $p$-value $<0.05$ (Supplementary Table 15).
Interestingly, interactions were also frequent within the region between 109 and $138 \mathrm{Mb}$, and more TADs and high eigenvector values were observed. These delineated compartments in $\mathrm{Hi}-\mathrm{C}^{47}$ and indicated that both the amplified region and an adjacent region had both frequent intrachromosomal interactions and frequent contacts, and it seemed that the entire $5 q$ region was upregulated by the amplification mechanism.

To compare the computed intrachromosomal interactions between MTX-resistant HT-29 cells and control samples, the difference in adjusted interaction frequencies (adjusted $M$ ) at $500 \mathrm{~kb}$ resolution and corresponding $p$-values were analyzed, and differentially interacting genomic regions at chromosome 5 were identified in the amplified region ( $p$-value $<0.05$; Fig. $5 b$ ). The adjusted $M$ values were lower in the region from 109 to $138 \mathrm{Mb}$ than in the amplified region, but this region still had a significant $\mathrm{p}$-value and a higher level of interactions compared to those in other positions. Therefore, the chromosomal structure, from the start position of the amplified unit $(80 \mathrm{Mb})$ to its endpoint on $5 \mathrm{q}$, could involve a complex network of spatial contacts to accommodate the gene amplification. 


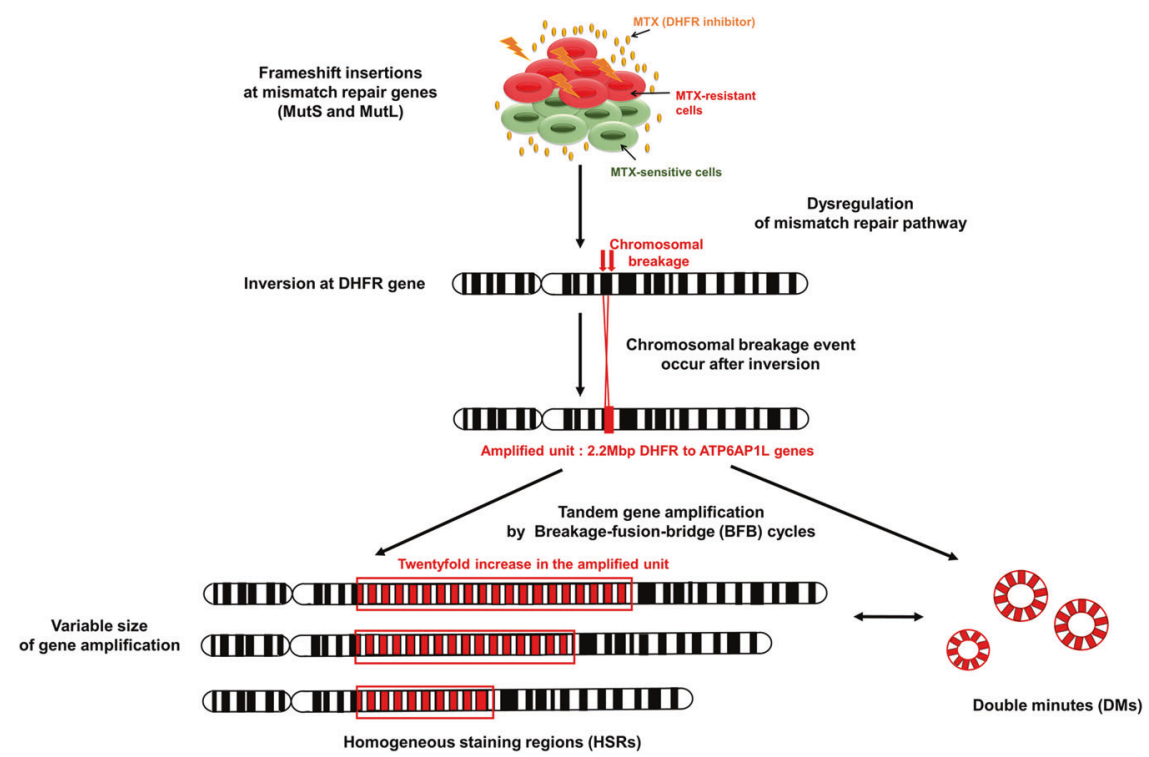

Fig. 6 The mechanism of tandem gene amplification in the presence of MTX. Frameshift insertions in mismatch repair family genes (mutS and mutL homologs) can initiate the dysregulation of mismatch repair pathways. A chromosomal breakage event occurs after the emergence of an inversion at DHFR. Those breaks that occurred between DHFR and ATP6AP1L $(2.2 \mathrm{Mb})$ were involved in producing the amplified unit. Finally, tandem amplifications of variable size can be achieved via breakage fusion-bridge (BFB) cycles.

The relative copy number of each chromosome was estimated from $\mathrm{Hi}-\mathrm{C}$ data using chromosome 2 as a reference. Specifically, the relative copy number of chromosome 5 was significantly higher in MTX-resistant HT-29 cells than in control cells over all chromosomes except for chromosome 17, which also had a stretched structure similar to that observed for chromosome 5 (Supplementary Fig. 15).

In addition, we observed chromosomal rearrangements, such as those that would result in DMs of extrachromosomal DNA stretches that harbored amplified oncogenes that were involved in drug resistance. These rearrangements were detected in the amplified region via $\mathrm{Hi}-\mathrm{C}$ and were not observed in the FISH data. $\mathrm{Hi}-\mathrm{C}$ data were utilized because they could reveal unforeseen chromosomal rearrangements and CNV in highly amplified regions. However, this result should be confirmed using other techniques, since it was not clear how to distinguish between DMs and HSRs due to their similar structure.

\section{The mechanisms underlying tandem gene amplification}

Using Censor, which identifies TEs, we analyzed the TEs, and we detected 46 TEs in the MTX-resistant sample (Supplementary Table 16$)^{29}$. LTRs $(43 \%$, total insertion: 20$)$, NonLTR/SINE/SINE1s, including Alu (8\%, total insertions: 4), and NonLTR/L1s, including LINE-1 (13\%, total insertions: 6), were detected. The detected retrotransposon insertions could promote structural instability, and Alu insertion in mismatch repair genes could affect recombination, deletions, and insertions throughout the genome, as previously reported ${ }^{48}$. In addition, LTRs (long terminal repeats) can alter alternative splicing patterns and polyadenylation signals. The L1ME3C_3 end of non-LTR/L1, which is a tandem gene repeat unit, was detected on RASGFR2 of chromosome 5. However, it was not found at the site of a structural variant such as an inversion, which was the starting position of gene amplification. Therefore, we concluded that LINE-1 (L1ME3C_3end) was inserted into the RASGFR2 gene but that this insertion was not the event that triggered chromosomal breakage ${ }^{49,50}$.

We have developed experimental and computational workflows to detect and analyze the mechanisms underlying gene amplification in MTX-resistant HT-29 cells through breakage-fusion-bridge (BFB) cycles, as previously reported ${ }^{51}$ (Fig. 6). Before gene amplification occurred, frameshift insertions in $M S H$ and $M L H$ genes across several chromosomes were caused by MTX toxicity, which resulted in a genetic predisposition to gene amplification and dysregulation of mismatch repair pathways in the presence of MTX $^{52,53}$.

After the malfunctioning of mismatch repair mechanisms in the presence of MTX, chromosomal breakages occurred at the DHFR gene on chromosome 5 because of the inverted repeat at the start position of the gene. In addition, inverted repeats from the DHFR gene to ATP6AP1L (2.2 Mb) were involved in producing the amplified unit. The endpoint of the amplified unit had the same inverted repeat, which could indicate the end position of the gene amplification. However, it is still unknown how the amplification of these specific genes was selected for and what factors are involved in the gene amplification mechanism.

Finally, variable sizes of amplified regions leading to HSRs could be produced via BFB cycles, and the unstable HSRs could be occasionally transformed into either a HSR fragment of a different size or DMs, accompanying inversions at the endpoints. Overall, the coamplification of MSH3 and DHFR and frameshift mutations in $M S H$ and $M L H$ continuously affected genetic instability and enhanced resistance to methotrexate.

\section{DISCUSSION}

Recent developments in NGS technologies have enabled the generation of long reads $(\sim 10 \mathrm{~kb})$ and are able to produce enormous amounts of genomic information at base-level resolution. Such detailed data can facilitate the analysis of uncharacterized regions that cannot be studied using other technologies ${ }^{54,55}$.

Here, complicated cancer genomes and abnormal chromosome structures were detected and analyzed using a combination of advanced technologies, including PacBio SMRT, optical mapping, and $\mathrm{Hi}-\mathrm{C}$ analysis, as well as short-read sequencing and FISH. An integrative framework was also used to detect and interpret the structural variations in MTX-resistant HT-29 cells. This analysis focused on a large repeat region and complex DNA segments. Finally, the amplified region was characterized and evaluated by size and relevant genetic defects, and potential gene amplification mechanisms were suggested.

To analyze the complicated repetitive sequences involved in gene amplification in the presence of MTX, several MTX-resistant 
clones were generated from an HT-29 cell line and selected by increasing the MTX concentration in an MTX sensitization procedure. When DHFR gene amplification was detected in several clones via FISH, the cells in each clone had a very large variation in the $5 q 12$ region. DHFR was amplified in a small portion of cells, given that tumor cells responded heterogeneously to MTX. Thus, the effects of the drug can be dissimilar, even when cells are grown under the same conditions ${ }^{56,57}$. This could be associated with bioinformatics difficulties in accurately defining amplification patterns and repetitive sequences, in both previous and future studies.

To overcome the analysis limitations posed by heterogeneous amplification patterns, DHFR amplification patterns were analyzed in specific, optimized clones that contained 96\% DHFRamplified cells. Approximately $75 \%$ of the cells in the clone converged to a homogeneous DHFR amplification pattern. After clone optimization, the amplified unit and tandem gene amplification of 11 genes, from the DHFR gene to the ATP6AP1L gene on chr5 (2.2 Mbp), were characterized on the basis of longrange genomic information obtained from long-read sequencing and optical genome mapping. The amplified unit had high coverage $(\sim 197 X)$ compared to the control $(\sim 10 X)$. This implied that the amplified region was tandemly amplified by $\sim 20$-fold compared to the original sequence, which was confirmed by gene expression analysis demonstrating high levels of transcripts of these genes and their splice variants. Genes within the amplified unit were highly overexpressed, exhibiting 5 -fold to 122 -fold increases in expression compared to that of the control. The variability of these expression patterns could reflect the complexity of splicing patterns, even if these genes were amplified simultaneously.

Furthermore, inversions at both the start and endpoints of the amplified unit were detected in long-read sequencing data, and this structural variant was cross-validated via optical genome mapping. Previous studies have also shown that inversions can lead to the initiation of gene amplification by stimulating chromosomal breakage. In addition, such a phenomenon could represent a transformation from circular DNA segment DMs to intrachromosomal HSRs ${ }^{58,59}$.

Using $\mathrm{Hi}-\mathrm{C}$ analysis, we identified high levels of intrachromosomal interactions within the amplified region and significant interactions at the novel TADs. An increase in long-range chromosomal interactions from 109 to $138 \mathrm{Mbp}$, compared with the amplified region, was also unexpectedly detected. The amplified unit in the $\mathrm{Hi}-\mathrm{C}$ analysis exactly matched that identified by the long-range genomic information and RNA sequencing results. This could indicate that the amplified position was compactly packed such that the amplified sequences had frequent contact with each other, which could reflect the chromatin architecture involved in gene amplification ${ }^{60}$.

In addition, novel frameshift insertions in $M S H$ and $M L H$ were identified in the MTX-resistant sample, which could play an important role in the rapid progression of gene amplification and MTX resistance. In this vein, microsatellite instability (MSI), i.e., a marker of deficient DNA mismatch repair in colon cancer, was tested to evaluate the status of MMR in MTX-resistant HT-29 cells. However, there was no significant difference in MSI between MTXresistant cells and the control ${ }^{61}$.

Taken together, these findings suggest that DHFR is likely not the only target of the MTX-associated mechanism, since 11 previously unimplicated genes were tandemly amplified between DHFR and ATP6AP1L, and the expanded region on the $5 q$ arm over the amplified region interacted with other amplified genes. The gene in the amplified unit with the highest expression was RASGRF2, which exhibited a 122 -fold increase compared with the control. Conversely, DHFR exhibited only a fivefold increase compared with the control. Therefore, more investigations are needed to target and assess the role of RASGRF2 in MTX-related gene amplification. This gene might utilize the DHFR promoter to achieve its high expression in the presence of MTX.

This implied that MSI did not affect genetic instability and the entire MMR system over whole chromosomes, whereas MTX toxicity could induce mutations in $M M R$ genes as well as genetic predisposition to amplification on chromosome 5 in MTX-resistant HT-29 cells through the insertion of adenine or thymine nucleotides into $M S H$ and $M L H$ genes. This points to a possible tandem gene amplification mechanism that progresses through BFB cycles. In addition, it was possible that BFB cycles under lowlevel MTX drug selection might generate ecDNAs and chromothripsis, as previously reported ${ }^{62}$. However, we estimate that this is less likely, since no DMs were detected by either FISH or HiC-trans.

Further studies are needed to identify whether a frameshift insertion in MSH3 might be caused by coamplification of DHFR and other frameshift insertions that result in the malfunctioning of mutS homologs and hypermutability in the presence of significant MTX $^{63,64}$. However, frameshift insertions in MSH and MLH could not be generated by the gene amplification process, since all of the mutated genes, except for $M S H 3$, are located outside of the amplified unit. Therefore, the frameshift mutations in each gene should have had a different cause in the presence of MTX toxicity as well as different impacts on MTX-resistant HT-29 cells.

In addition, the mismatch location is recognized by two mutS homologs, mutS-alpha (MSH2 and MSH6), which is known to facilitate the repair of single-nucleotide mismatches, and mutSbeta (MSH2 and MSH3), which is known to assist in the repair of large indels ${ }^{65,66}$. Such mutS homologs should require mutL homologs (MLH1 and PMS2) for binding to a recognition site. Therefore, mutations in mutS and mutL homologs could prevent the repair of single-nucleotide mismatches and large indels in MTX-resistant HT-29 cells. The prevention of mutations and inversions in mutS and mutL homologs could increase cell sensitivity to MTX and inhibit gene amplification.

Although this study has several limitations, our findings may provide new insight into the mechanisms underlying the amplification process and evolution of MTX resistance in colon cancer and leukemia. Moreover, the use of $\mathrm{Hi}-\mathrm{C}$ data to detect unforeseen chromosomal rearrangements such as DMs and HSRs has been shown to be promising for future analyses.

Most importantly, additional validation of the identified SVs and repetitive sequences is required to interpret the entire sequence of events involved in the gene amplification mechanism and the impact of amplification on MTX resistance. This information will provide clues to understanding how cancers adapt to drugs. Finally, our findings will bolster clinical cancer studies and inform diagnoses as well as the management and treatment of various cancers and provide in-depth guidance toward pharmacologic targets for anticancer drugs as well as personalized medicine.

\section{DATA AVAILABILITY}

The datasets generated and/or analyzed in the current study are available from the corresponding author on reasonable request. Supplementary information accompanies the manuscript on the Experimental \& Molecular Medicine website (http:// www.nature.com/emm/). The preprint is available on BioRxiv (https://www.biorxiv org/content/10.1101/2020.02.26.965814v1.abstract) ${ }^{67}$.

\section{REFERENCES}

1. Schwab, M. Oncogene amplification in solid tumors. Semin. Cancer Biol. 9, 319-325 (1999).

2. Marotta, M. et al. A common copy-number breakpoint of ERBB2 amplification in breast cancer colocalizes with a complex block of segmental duplications. Breast Cancer Res. 14, R150 (2012).

3. Mathew, P. et al. Detection of MYCN gene amplification in neuroblastoma by fluorescence in situ hybridization: a pediatric oncology group study. Neoplasia $\mathbf{3}$ 105-109 (2001).

4. Kaufman, R. J. \& Schimke, R. T. Amplification and loss of dihydrofolate reductase genes in a Chinese hamster ovary cell line. Mol. Cell Biol. 1, 1069-1076 (1981) 
5. Ohta, J. I. et al. Fluorescence in situ hybridization evaluation of c-erbB-2 gene amplification and chromosomal anomalies in bladder cancer. Clin. Cancer Res. 7, 2463-2467 (2001).

6. Dacic, S. et al. Significance of EGFR protein expression and gene amplification in non-small cell lung carcinoma. Am. J. Clin. Pathol. 125, 860-865 (2006).

7. Kamel, H. F. M. \& Al-Amodi, H. Exploitation of gene expression and cancer biomarkers in paving the path to era of personalized medicine. Genomics Proteom. Bioinforma. 15, 220-235 (2017).

8. Schimke, R. T. Gene amplification in cultured animal cells. Cell 37, 705-713 (1984).

9. Kang, J. U. Characterization of amplification patterns and target genes on the short arm of chromosome 7 in early-stage lung adenocarcinoma. Mol. Med. Rep. 8, 1373-1378 (2013).

10. Treangen, T. J. \& Salzberg, S. L. Repetitive DNA and next-generation sequencing: computational challenges and solutions. Nat. Rev. Genet. 13, 36-46 (2011).

11. Schneeberger, K. et al. Simultaneous alignment of short reads against multiple genomes. Genome Biol. 10, R98 (2009).

12. Nattestad, $M$. et al. Complex rearrangements and oncogene amplifications revealed by long-read DNA and RNA sequencing of a breast cancer cell line. Genome Res. 28, 1126-1135 (2018).

13. Amarasinghe, S. L. et al. Opportunities and challenges in long-read sequencing data analysis. Genome Biol. 21, 30 (2020).

14. Brown, P. C., Tlsty, T. D. \& Schimke, R. T. Enhancement of methotrexate resistance and dihydrofolate reductase gene amplification by treatment of mouse 3T6 cells with hydroxyurea. Mol. Cell Biol. 3, 1097-1107 (1983).

15. Goker, E. et al. Amplification of the dihydrofolate reductase gene is a mechanism of acquired resistance to methotrexate in patients with acute lymphoblastic leukemia and is correlated with p53 gene mutations. Blood 86, 677-684 (1995).

16. Morales, C., Ribas, M., Aiza, G. \& Peinado, M. A. Genetic determinants of methotrexate responsiveness and resistance in colon cancer cells. Oncogene 24, 6842-6847 (2005).

17. Morales, C. et al. Dihydrofolate reductase amplification and sensitization to methotrexate of methotrexate-resistant colon cancer cells. Mol. Cancer Ther. 8 , 424-432 (2009).

18. Seo, J. S. et al. Whole exome and transcriptome analyses integrated with microenvironmental immune signatures of lung squamous cell carcinoma. Cancer Immunol. Res. 6, 848-859 (2018).

19. McKenna, A. et al. The Genome Analysis Toolkit: a MapReduce framework for analyzing next-generation DNA sequencing data. Genome Res. 20, 1297-1303 (2010).

20. Seo, J. S. et al. The transcriptional landscape and mutational profile of lung adenocarcinoma. Genome Res. 22, 2109-2119 (2012).

21. Shen, $\mathrm{S}$. et al. rMATS: robust and flexible detection of differential alternative splicing from replicate RNA-Seq data. Proc. Natl Acad. Sci. USA 111, E5593-E5601 (2014).

22. Sedlazeck, F. J. et al. Accurate detection of complex structural variations using single-molecule sequencing. Nat. Methods 15, 461-468 (2018).

23. Haghshenas, E., Sahinalp, S. C. \& Hach, F. lordFAST: sensitive and Fast Alignment Search Tool for LOng noisy Read sequencing Data. Bioinformatics 35, 20-27 (2019).

24. Seo, J. S. et al. De novo assembly and phasing of a Korean human genome. Nature 538, 243-247 (2016).

25. Lieberman-Aiden, E. et al. Comprehensive mapping of long-range interactions reveals folding principles of the human genome. Science 326, 289-293 (2009).

26. Servant, N. et al. HiC-Pro: an optimized and flexible pipeline for $\mathrm{Hi}-\mathrm{C}$ data processing. Genome Biol. 16, 259 (2015).

27. Chakraborty, A. \& Ay, F. Identification of copy number variations and translocations in cancer cells from Hi-C data. Bioinformatics 34, 338-345 (2017).

28. Stansfield, J. C., Cresswell, K. G., Vladimirov, V. I. \& Dozmorov, M. G. HiCcompare: an R-package for joint normalization and comparison of $\mathrm{HI}-\mathrm{C}$ datasets. BMC Bioinforma. 19, 279 (2018).

29. Kohany, O., Gentles, A. J., Hankus, L. \& Jurka, J. Annotation, submission and screening of repetitive elements in Repbase: RepbaseSubmitter and Censor. BMC Bioinforma. 7, 474 (2006).

30. Meng, X. et al. Novel role for non-homologous end joining in the formation of double minutes in methotrexate-resistant colon cancer cells. J. Med. Genet. 52, 135-144 (2015).

31. Schildhaus, H. U. et al. Definition of a fluorescence in-situ hybridization score identifies high- and low-level FGFR1 amplification types in squamous cell lung cancer. Mod. Pathol. 25, 1473-1480 (2012).

32. Schimke, R. T. Gene amplification and drug resistance. Sci. Am. 243, 60-69 (1980).

33. Ashktorab, H. et al. Distinct genetic alterations in colorectal cancer. PLOS ONE 5, e8879 (2010).

34. Reams, A. B. \& Roth, J. R. Mechanisms of gene duplication and amplification. Cold Spring Harb. Perspect. Biol. 7, a016592 (2015).

35. Tanaka, H., Tapscott, S. J., Trask, B. J. \& Yao, M. C. Short inverted repeats initiate gene amplification through the formation of a large DNA palindrome in mammalian cells. Proc. Natl Acad. Sci. USA 99, 8772-8777 (2002).
36. Edelmann, W. et al. The DNA mismatch repair genes Msh3 and Msh6 cooperate in intestinal tumor suppression. Cancer Res. 60, 803-807 (2000).

37. Karahan, B., Argon, A., Yildirim, M. \& Vardar, E. Relationship between MLH-1, MSH2, PMS-2,MSH-6 expression and clinicopathological features in colorectal cancer. Int. J. Clin. Exp. Pathol. 8, 4044-4053 (2015).

38. Edelmann, W. et al. Mutation in the mismatch repair gene Msh6 causes cancer susceptibility. Cell 91, 467-477 (1997).

39. Silva, F. C., Valentin, M. D., Ferreira Fde, O., Carraro, D. M. \& Rossi, B. M. Mismatch repair genes in Lynch syndrome: a review. Sao Paulo Med. J. 127, 46-51 (2009).

40. Kariola, R., Raevaara, T. E., Lonnqvist, K. E. \& Nystrom-Lahti, M. Functional analysis of MSH6 mutations linked to kindreds with putative hereditary non-polyposis colorectal cancer syndrome. Hum. Mol. Genet. 11, 1303-1310 (2002).

41. Drummond, J. T., Genschel, J., Wolf, E. \& Modrich, P. DHFR/MSH3 amplification in methotrexate-resistant cells alters the hMutSalpha/hMutSbeta ratio and reduces the efficiency of base-base mismatch repair. Proc. Natl Acad. Sci. USA 94, 10144-10149 (1997).

42. Guo, Y., Bao, Y., Ma, M. \& Yang, W. Identification of key candidate genes and pathways in colorectal cancer by integrated bioinformatical analysis. Int. J. Mol. Sci. 18, 722 (2017)

43. Yang, X. R., Xiong, Y., Duan, H. \& Gong, R. R. Identification of genes associated with methotrexate resistance in methotrexate-resistant osteosarcoma cell lines. J. Orthop. Surg. Res. 10, 136 (2015).

44. Uchiyama, H. et al. Cyclin-dependent kinase inhibitor SU9516 enhances sensitivity to methotrexate in human T-cell leukemia Jurkat cells. Cancer Sci. 101, 728-734 (2010)

45. O'Brien, V. \& Brown, R. Signalling cell cycle arrest and cell death through the MMR System. Carcinogenesis 27, 682-692 (2006).

46. Harewood, $\mathrm{L}$. et al. $\mathrm{Hi}-\mathrm{C}$ as a tool for precise detection and characterisation of chromosomal rearrangements and copy number variation in human tumours. Genome Biol. 18, 125 (2017).

47. Rao, S. S. et al. A 3D map of the human genome at kilobase resolution reveals principles of chromatin looping. Cell 159, 1665-1680 (2014)

48. Shademan, M. et al. Promoter methylation, transcription, and retrotransposition of LINE-1 in colorectal adenomas and adenocarcinomas. Cancer Cell Int. 20, 426 (2020).

49. Wheeler, T. J. et al. Dfam: a database of repetitive DNA based on profile hidden Markov models. Nucleic Acids Res. 41, D70-D82 (2013).

50. Lynch-Sutherland, C. F., Chatterjee, A., Stockwell, P. A., Eccles, M. R. \& Macaulay, E. C. Reawakening the developmental origins of cancer through transposable elements. Front. Oncol. 10, 468 (2020).

51. Matsui, A., Ihara, T., Suda, H., Mikami, H. \& Semba, K. Gene amplification: mechanisms and involvement in cancer. Biomol. Concepts 4, 567-582 (2013).

52. Singer, M. J., Mesner, L. D., Friedman, C. L., Trask, B. J. \& Hamlin, J. L. Amplification of the human dihydrofolate reductase gene via double minutes is initiated by chromosome breaks. Proc. Natl Acad. Sci. USA 97, 7921-7926 (2000).

53. Verhaak, R. G. W., Bafna, V. \& Mischel, P. S. Extrachromosomal oncogene amplification in tumour pathogenesis and evolution. Nat. Rev. Cancer 19, 283-288 (2019).

54. Kulkarni, P. \& Frommolt, P. Challenges in the setup of large-scale next-generation sequencing analysis workflows. Comput. Struct. Biotechnol. J. 15, 471-477 (2017).

55. Sedlazeck, F. J., Lee, H., Darby, C. A. \& Schatz, M. C. Piercing the dark matter: bioinformatics of long-range sequencing and mapping. Nat. Rev. Genet. 19, 329-346 (2018)

56. Lengauer, C., Kinzler, K. W. \& Vogelstein, B. Genetic instabilities in human cancers. Nature 396, 643-649 (1998).

57. Stapf, M., Pompner, N., Teichgraber, U. \& Hilger, I. Heterogeneous response of different tumor cell lines to methotrexate-coupled nanoparticles in presence of hyperthermia. Int. J. Nanomed. 11, 485-500 (2016).

58. L'Abbate, A. et al. Genomic organization and evolution of double minutes/ homogeneously staining regions with MYC amplification in human cancer. Nucleic Acids Res. 42, 9131-9145 (2014).

59. Cheng, C. et al. Whole-Genome Sequencing Reveals Diverse Models of Structural Variations in Esophageal Squamous Cell Carcinoma. Am. J. Hum. Genet. 98, 256-274 (2016).

60. Carty, M. et al. An integrated model for detecting significant chromatin interactions from high-resolution Hi-C data. Nat. Commun. 8, 15454 (2017).

61. Kawakami, H., Zaanan, A. \& Sinicrope, F. A. Microsatellite instability testing and its role in the management of colorectal cancer. Curr. Treat. Options Oncol. 16, 30 (2015).

62. Shoshani, O. et al. Chromothripsis drives the evolution of gene amplification in cancer. Nature 591, 137-141 (2021).

63. Matheson, E. C., Hogarth, L. A., Case, M. C., Irving, J. A. \& Hall, A. G. DHFR and MSH3 co-amplification in childhood acute lymphoblastic leukaemia, in vitro and in vivo. Carcinogenesis 28, 1341-1346 (2007).

64. Marra, G. et al. Mismatch repair deficiency associated with overexpression of the MSH3 gene. Proc. Natl Acad. Sci. USA 95, 8568-8573 (1998). 
65. Willems, R. J. et al. Mutations in the DNA mismatch repair proteins MutS and MutL of oxazolidinone-resistant or -susceptible Enterococcus faecium. Antimicrob. Agents Chemother. 47, 3061-3066 (2003).

66. Junop, M. S., Yang, W., Funchain, P., Clendenin, W. \& Miller, J. H. In vitro and in vivo studies of MutS, MutL and MutH mutants: correlation of mismatch repair and DNA recombination. DNA Repair 2, 387-405 (2003).

67. Kim, A., Shin, J.-Y. \& Seo, J.-S. Genomic and transcriptomic analyses reveal a tandem amplification unit of 11 genes and mutations of mismatch repair genes in methotrexate-resistant HT-29 cells. Preprint at https://www.biorxiv.org/ content/10.1101/2020.02.26.965814v1.full (2020)

\section{ACKNOWLEDGEMENTS}

This work has been supported by Macrogen Inc. (grant no. MGR17-04).

\section{AUTHOR CONTRIBUTIONS}

J.-S.S. and J.-Y.S. conceived and designed the experiments. A.K. conducted the experiment by preparing the MTX-resistant HT-29 cells and performing DNA and RNA extraction. J.-Y.S. performed PacBio long-read seq and whole-transcriptome seq experiments. A.K. performed sequencing data processing, bioinformatics and statistical analyses. J.-S.S., A.K., and J.-Y.S. wrote the manuscript.

\section{COMPETING INTERESTS}

The authors declare no competing interests.

\section{ADDITIONAL INFORMATION}

Supplementary information The online version contains supplementary material available at https://doi.org/10.1038/s12276-021-00668-x.

Correspondence and requests for materials should be addressed to Jeong-Sun Seo.

Reprints and permission information is available at http://www.nature.com/ reprints

Publisher's note Springer Nature remains neutral with regard to jurisdictional claims in published maps and institutional affiliations.

(i) Open Access This article is licensed under a Creative Commons Attribution 4.0 International License, which permits use, sharing, adaptation, distribution and reproduction in any medium or format, as long as you give appropriate credit to the original author(s) and the source, provide a link to the Creative Commons license, and indicate if changes were made. The images or other third party material in this article are included in the article's Creative Commons license, unless indicated otherwise in a credit line to the material. If material is not included in the article's Creative Commons license and your intended use is not permitted by statutory regulation or exceeds the permitted use, you will need to obtain permission directly from the copyright holder. To view a copy of this license, visit http://creativecommons. org/licenses/by/4.0/.

(c) The Author(s) 2021 